\title{
AN INITIAL VALUE METHOD FOR SINGULARLY PERTURBED SYSTEM OF REACTION-DIFFUSION TYPE DELAY DIFFERENTIAL EQUATIONS
}

\author{
V. SUBBURAYAN ${ }^{1}$ AND N. RAMANUJAM ${ }^{2}$ \\ ${ }^{1}$ Department of Mathematics, SRM University, Kattankulathur-603203, Kancheepuram, \\ INDIA. \\ E-mail address: suburayan123@gmail.com \\ ${ }^{2}$ Department of Mathematics, Bharathidasan University, TiruchirapPalli-620024, India \\ E-mail address: matram2k3@gmail.com
}

\begin{abstract}
In this paper an asymptotic numerical method named as Initial Value Method (IVM) is suggested to solve the singularly perturbed weakly coupled system of reaction-diffusion type second order ordinary differential equations with negative shift (delay) terms. In this method, the original problem of solving the second order system of equations is reduced to solving eight first order singularly perturbed differential equations without delay and one system of difference equations. These singularly perturbed problems are solved by the second order hybrid finite difference scheme. An error estimate for this method is derived by using supremum norm and it is of almost second order. Numerical results are provided to illustrate the theoretical results.
\end{abstract}

\section{INTRODUCTION}

Modeling automatic engines or physiological systems often involves the idea of control because feedback is used in order to maintain a stable state. But much of this feedback require a finite time to sense information and react to it. A popular way to describe this process is to formulate a Delay Differential Equations or Differential Difference Equations (DDEs) where the evolution of a dependent variable is a function of time which depends not only current time but also earlier time. For example, a modeling of water flows at a uniform rate from the faucet to the shower head [1], etc.

A subclass of these equations consist of singularly perturbed ordinary differential equations with a delay, that is an ordinary differential equation in which the highest derivative is multiplied by a small parameter $\varepsilon$ and involving at least one delay (negative shift) term. These kinds of equations arise frequently in the mathematical modeling of various practical phenomena, for example, in the modeling of the human pupil-light reflex [2], first-exit time problem [3], the study of bistable devices [4] and variational problems in control theory [5], etc.

Received by the editors June 27, 2013; Revised August 6 2013; Accepted in revised form August 102013.

2010 Mathematics Subject Classification. 34K10, 34K26, 34K28.

Key words and phrases. Weakly coupled system, Reaction-diffusion type equations, Initial value method, Shishkin mesh, Delay, Negative shift. 
It is well known that standard discretization methods for solving singular perturbation problems for differential equations are sometimes unstable and fail to give accurate results when the perturbation parameter $\varepsilon$ is small. Therefore, it is important to develop suitable numerical methods to solve these types of equations, whose accuracy does not depend on the parameter $\varepsilon$, that is methods which are uniformly convergent with respect to the parameter $\varepsilon$. For more details in this direction one may refer to $[6,7,8,9]$.

In $[10,11,12]$ and the references therein the authors presented various numerical methods for the following Singularly Perturbed Delay Differential Equations (SPDDEs)

$$
\begin{aligned}
& \varepsilon y^{\prime \prime}+a(x) y^{\prime}(x-\delta)+b(x) y^{\prime}(x)+c(x) y(x-\delta)+e(x) y(x)=f(x), \\
& y(x)=\phi(x), x \in[-\delta, 0], y(1)=\gamma, \delta=o(\varepsilon) .
\end{aligned}
$$

In fact, for various combinations of conditions on the coefficients $a, b, c$ and $e$ (for an example $a<0, b=c=0, e<0$ [11]) they suggested suitable numerical methods. In all these methods first they applied Taylor's expansion for $y^{\prime}(x-\delta)$ or $y(x-\delta)$ and reduced the DDEs to DEs. For the resulting DEs they applied standard numerical methods available in the literature. Following this procedure some authors $[13,14]$ suggested numerical methods to the following Boundary Value Problem (BVP):

$$
\begin{aligned}
& \varepsilon y^{\prime \prime}+c(x) y(x-\delta)+d(x) y(x+\eta)+e(x) y(x)=f(x), \\
& y(x)=\phi(x), x \in[-\delta, 0], y(x)=\gamma(x), x \in[1,1+\eta],
\end{aligned}
$$

where $\delta=o(\varepsilon), \eta=o(\varepsilon)$.

A few authors [15] considered the above DDE (1.1)-(1.2) with $a \neq 0, b=0, c=0$ and suggested numerical methods without applying Taylor's expansion, that is, reducing the DDE to $\mathrm{DE}$.

Using the Taylor's series expansion procedure as mentioned above and Newton's quasi linearization process some authors $[16,17]$ solved nonlinear problems numerically.

Subburayan and Ramanujam [18, 19] suggested a numerical method namely initial value technique for the following BVP

$$
\left\{\begin{array}{l}
-\varepsilon u^{\prime \prime}+a(x) u^{\prime}(x)+b(x) u(x)+c(x) u(x-1)=f(x), x \in(0,1) \cup(1,2), \\
u(x)=\phi(x),[-1,0], u(2)=l,
\end{array}\right.
$$

where $a$ can be either continuous throughout the domain $[0,2]$ or continuous except at $x=1$. The motivation for the consideration of the above SPDDE (1.5) and below (2.1) has come from the paper of Lange and Miura [20]. In the present paper, as said in the abstract, a numerical method named as IVM is suggested to solve the following singularly perturbed weakly coupled system of reaction-diffusion type second order ordinary differential equations with negative shift (2.1).

The present paper is organized as follows. In Section 2, the problem under study with continuous source term is stated. A maximum principle for the differential-difference operators $P_{i}, i=1,2$ defined in Section 2 is established in Section 3. Further a stability result is derived. An asymptotic expansion approximation for the solution of the current problem is derived in 
Section 4. The present numerical method namely the Initial Value Method (IVM) is described in Section 5 and an error estimate is derived in Section 6. Section 7 is devoted for discontinuous source term. Section 8 presents numerical results. The paper is concluded with a discussion (Section 9).

\section{Statement of the Problem}

Throughout the paper, we assume that $\sqrt{\varepsilon} \leq C N^{-1}$ and $C$ and $C_{1}$ denote generic positive constants independent of the singular perturbation parameter $\varepsilon$ and the discretization parameter $N$ of the discrete problem. This condition is used to prove the Theorem 5.1 of this paper. The supremum norm is used for studying the convergence of the numerical solution to the exact solution of a singular perturbation problem: $\|\phi\|_{D}=\sup _{x \in D}|\phi(x)|$.

Motivated by the work of [20], we consider the following Boundary Value Problem (BVP) for SPDDE.

Find $\bar{u}=\left(u_{1}, u_{2}\right), u_{1}, u_{2} \in Y=C^{0}(\bar{\Omega}) \cap C^{1}(\Omega) \cap C^{2}\left(\Omega^{*}\right)$ such that

$$
\left\{\begin{array}{l}
-\varepsilon u_{1}^{\prime \prime}(x)+a_{1}(x) u_{1}(x)+\sum_{k=1}^{2} b_{1 k}(x) u_{k}(x-1)=f_{1}(x), x \in \Omega^{*}, \\
-\varepsilon u_{2}^{\prime \prime}(x)+a_{2}(x) u_{2}(x)+\sum_{k=1}^{2} b_{2 k}(x) u_{k}(x-1)=f_{2}(x), x \in \Omega^{*}, \\
u_{1}(x)=\phi_{1}(x), x \in[-1,0], u_{1}(2)=l_{1}, \\
u_{2}(x)=\phi_{2}(x), x \in[-1,0], u_{2}(2)=l_{2},
\end{array}\right.
$$

where $0<\varepsilon \ll 1, a_{i}(x) \geq \alpha_{i}>\alpha>0, b_{i j}(x) \leq 0, i=1,2, j=1,2,0 \geq b_{i 1}(x)+b_{i 2}(x) \geq$ $\beta_{i}, i=1,2, \alpha_{i}+\beta_{i} \geq \alpha>0$, and $a_{i}, b_{i j}, f_{i} i=1,2, j=1,2$ are sufficiently smooth functions on $\bar{\Omega}, \Omega=(0,2), \bar{\Omega}=[0,2], \Omega^{*}=\Omega^{-} \cup \Omega^{+}, \Omega^{-}=(0,1), \Omega^{+}=(1,2)$ and $\phi_{i}, i=1,2$ are smooth on $[-1,0]$.

The above problem is equivalent to

$$
\begin{gathered}
P_{1} \bar{u}(x):=\left\{\begin{array}{l}
-\varepsilon u_{1}^{\prime \prime}(x)+a_{1}(x) u_{1}(x)=f_{1}(x)-\sum_{k=1}^{2} b_{1 k}(x) \phi_{k}(x-1), x \in \Omega^{-}, \\
-\varepsilon u_{1}^{\prime \prime}(x)+a_{1}(x) u_{1}(x)+\sum_{k=1}^{2} b_{1 k}(x) u_{k}(x-1)=f_{1}(x), x \in \Omega^{+},
\end{array}\right. \\
P_{2} \bar{u}(x):=\left\{\begin{array}{l}
-\varepsilon u_{2}^{\prime \prime}(x)+a_{2}(x) u_{2}(x)=f_{2}(x)-\sum_{k=1}^{2} b_{2 k}(x) \phi_{k}(x-1), x \in \Omega^{-}, \\
-\varepsilon u_{2}^{\prime \prime}(x)+a_{2}(x) u_{2}(x)+\sum_{k=1}^{2} b_{2 k}(x) u_{k}(x-1)=f_{2}(x), x \in \Omega^{+},
\end{array}\right. \\
\left\{\begin{array}{l}
u_{1}(0)=\phi_{1}(0), u_{1}(1-)=u_{1}(1+), u_{1}^{\prime}(1-)=u_{1}^{\prime}(1+), u_{1}(2)=l_{1}, \\
u_{2}(0)=\phi_{2}(0), u_{2}(1-)=u_{2}(1+), u_{2}^{\prime}(1-)=u_{2}^{\prime}(1+), u_{2}(2)=l_{2},
\end{array}\right.
\end{gathered}
$$

where $u_{1}(1-)$ and $u_{1}(1+)$ denote the left and right limits of $u_{1}$ at $x=1$ and the similar expressions are true for other functions. 


\section{STABiLity Result}

The differential-difference operators $P_{i}, i=1,2$ defined in the above section satisfy the following maximum principle.

Theorem 3.1. (Maximum principle) Let $\bar{w}=\left(w_{1}, w_{2}\right), w_{1}, w_{2} \in C^{0}(\bar{\Omega}) \cap C^{2}\left(\Omega^{*}\right)$ be any function satisfying $w_{i}(0) \geq 0, w_{i}(2) \geq 0, P_{i} \bar{w}(x) \geq 0, \forall x \in \Omega^{*}$ and $w_{i}^{\prime}(1+)-w_{i}^{\prime}(1-)$ $=\left[w_{i}^{\prime}\right](1) \leq 0, i=1,2$. Then $w_{i}(x) \geq 0, \forall x \in \bar{\Omega}, i=1,2$.

Proof. Define $\bar{s}=\left(s_{1}, s_{2}\right)$, where

$$
s_{1}(x)=s_{2}(x)= \begin{cases}\frac{1}{8}+\frac{x}{2}, & x \in[0,1], \\ \frac{3}{8}+\frac{x}{4}, & x \in[1,2] .\end{cases}
$$

Note that $s_{i}(x)>0, \forall x \in \bar{\Omega}, P_{i} \bar{s}(x)>0, \forall x \in \Omega^{*}$ and $\left[s_{i}^{\prime}\right](1)<0, i=1,2$. Let

$$
\mu=\max \left\{\max _{x \in \bar{\Omega}}\left\{\frac{-w_{1}(x)}{s_{1}(x)}\right\}, \max _{x \in \bar{\Omega}}\left\{\frac{-w_{2}(x)}{s_{2}(x)}\right\}\right\} .
$$

Then there exists at least one $x_{0} \in \bar{\Omega}$ such that $w_{1}\left(x_{0}\right)+\mu s_{1}\left(x_{0}\right)=0$ or $w_{2}\left(x_{0}\right)+\mu s_{2}\left(x_{0}\right)=0$ or both and $w_{i}(x)+\mu s_{i}(x) \geq 0, \forall x \in \bar{\Omega}, i=1,2$. Without the loss of generality we assume that $w_{1}\left(x_{0}\right)+\mu s_{1}\left(x_{0}\right)=0$. Therefore the function $\left(w_{1}+\mu s_{1}\right)$ attains its minimum at $x=x_{0}$. Suppose the theorem does not hold true, then $\mu>0$.

Let $x_{0} \in \Omega^{-}$.

$$
0<P_{1}(\bar{w}+\mu \bar{s})\left(x_{0}\right)=-\varepsilon\left(w_{1}+\mu s_{1}\right)^{\prime \prime}\left(x_{0}\right)+a_{1}\left(x_{0}\right)\left(w_{1}+\mu s_{1}\right)\left(x_{0}\right) \leq 0 .
$$

It is a contradiction.

Similarly one can consider the case $x_{0} \in \Omega^{+}$and get a contradiction.

Let $x_{0}=1$.

$$
0 \leq\left[\left(w_{1}+\mu s_{1}\right)^{\prime}\right](1)=\left[w_{1}^{\prime}\right](1)+\mu\left[s_{1}^{\prime}\right](1)<0 .
$$

It is a contradiction.

Corollary 3.2. (Stability Result) Let $\bar{u}=\left(u_{1}, u_{2}\right), u_{1}, u_{2} \in Y$ be any function. Then

$$
\begin{array}{r}
\left|u_{j}(x)\right| \leq C \max \left\{\max _{i=1,2}\left\{\left|u_{i}(0)\right|\right\}, \max _{i=1,2}\left\{\left|u_{i}(2)\right|\right\}, \max _{i=1,2}\left\{\left\|P_{i} \bar{u}\right\|_{\Omega^{*}}\right\}\right\}, \\
\forall x \in \bar{\Omega}, j=1,2 .
\end{array}
$$

Proof. Let $C>0$ be a constant. Define $\bar{\psi}^{ \pm}=\left(\psi_{1}^{ \pm}, \psi_{2}^{ \pm}\right)$, where

$$
\begin{gathered}
\psi_{i}^{ \pm}(x)=C C_{1} s_{i}(x) \pm u_{i}(x), x \in \bar{\Omega}, i=1,2, \\
C_{1}=\max \left\{\max _{i=1,2}\left\{\left|u_{i}(0)\right|\right\}, \max _{i=1,2}\left\{\left|u_{i}(2)\right|\right\}, \max _{i=1,2}\left\{\left\|P_{i} \bar{u}\right\|_{\Omega^{*}}\right\}\right\} . \text { Then } \\
\psi_{i}^{ \pm}(0)=C C_{1} s_{i}(0) \pm u_{i}(0)>0 \text { and } \psi_{i}^{ \pm}(2)=C C_{1} s_{i}(2) \pm u_{i}(2)>0, i=1,2 \text { by a proper }
\end{gathered}
$$


choice of $C$.

Let $x \in \Omega^{-}$.

$$
P_{1} \bar{\psi}^{ \pm}(x)=C C_{1} P_{1} \bar{s}(x) \pm P_{1} \bar{u}(x) \geq \frac{C C_{1} \alpha_{1}}{8} \pm P_{1} \bar{u}(x) \geq 0,
$$

by a proper choice of $C$.

Similarly one can prove that, $P_{1} \bar{\psi}^{ \pm}(x) \geq 0$ in $\Omega^{+}$. Therefore $P_{1} \bar{\psi}^{ \pm}(x) \geq 0$ in $\Omega^{*}$. Similarly one can prove that $P_{2} \bar{\psi}^{ \pm}(x) \geq 0$ in $\Omega^{*}$. Further,

$$
\left[\psi_{i}^{ \pm^{\prime}}\right](1)=C C_{1}\left[s_{i}^{\prime}\right](1)+\left[u_{i}^{\prime}\right](1)<0, i=1,2
$$

by a proper choice of $C$.

Then by Theorem 3.1, we have $\psi_{i}^{ \pm}(x) \geq 0, x \in \bar{\Omega}, i=1,2$. Therefore

$\left|u_{j}(x)\right| \leq C \max \left\{\max _{i=1,2}\left\{\left|u_{i}(0)\right|\right\}, \max _{i=1,2}\left\{\left|u_{i}(2)\right|\right\}, \max _{i=1,2}\left\{\left\|P_{i} \bar{u}\right\|_{\Omega^{*}}\right\}\right\}, \forall x \in \bar{\Omega}, j=1,2$.

\section{AN ASYMPTOTIC EXPANSION}

In this section, an asymptotic expansion approximation for the solution of the problem (2.1) is constructed using the fundamental idea of WKB method [21].

Let $\bar{u}_{0}(x)=\left(u_{01}(x), u_{02}(x)\right), u_{01}, u_{02} \in C^{0}\left(\Omega^{*} \cup\{0,2\}\right)$ be the solution of the reduced problem of (2.1) given by

$$
\left\{\begin{array}{l}
a_{1}(x) u_{01}(x)+\sum_{k=1}^{2} b_{1 k}(x) u_{0 k}(x-1)=f_{1}(x), x \in \Omega^{*} \\
a_{2}(x) u_{02}(x)+\sum_{k=1}^{2} b_{2 k}(x) u_{0 k}(x-1)=f_{2}(x), x \in \Omega^{*} \\
u_{01}(x)=\phi_{1}(x), x \in[-1,0) \\
u_{02}(x)=\phi_{2}(x), x \in[-1,0)
\end{array}\right.
$$

and assumed that $\left\|u_{0 i}^{(2)}\right\|_{\Omega^{*}} \leq C, i=1,2$. Further, let $v_{1}, v_{2}, v_{3}, v_{4}, w_{1}, w_{2}, w_{3}$ and $w_{4}$ be the solutions of the following problems (4.2)-(4.9), respectively:

$$
\begin{gathered}
L_{1} v_{1}=\sqrt{\varepsilon} v_{1}^{\prime}(x)+\sqrt{a_{1}(x)} v_{1}(x)=0, x \in(0,2], v_{1}(0)=1, \\
L_{2} v_{2}=\sqrt{\varepsilon} v_{2}^{\prime}(x)-\sqrt{a_{1}(x)} v_{2}(x)=0, x \in[0,1), v_{2}(1)=1, \\
L_{1} v_{3}=\sqrt{\varepsilon} v_{3}^{\prime}(x)+\sqrt{a_{1}(x)} v_{3}(x)=0, x \in(1,2], v_{3}(1)=1, \\
L_{2} v_{4}=\sqrt{\varepsilon} v_{4}^{\prime}(x)-\sqrt{a_{1}(x)} v_{4}(x)=0, x \in[0,2), v_{4}(2)=1, \\
L_{3} w_{1}=\sqrt{\varepsilon} w_{1}^{\prime}(x)+\sqrt{a_{2}(x)} w_{1}(x)=0, x \in(0,2], w_{1}(0)=1, \\
L_{4} w_{2}=\sqrt{\varepsilon} w_{2}^{\prime}(x)-\sqrt{a_{2}(x)} w_{2}(x)=0, x \in[0,1), w_{2}(1)=1, \\
L_{3} w_{3}=\sqrt{\varepsilon} w_{3}^{\prime}(x)+\sqrt{a_{2}(x)} w_{3}(x)=0, x \in(1,2], w_{3}(1)=1, \\
L_{4} w_{4}=\sqrt{\varepsilon} w_{4}^{\prime}(x)-\sqrt{a_{2}(x)} w_{4}(x)=0, x \in[0,2), w_{4}(2)=1 .
\end{gathered}
$$


Now an asymptotic expansion approximation to the solution of the original problem (2.1) is given by

$$
\begin{aligned}
& u_{a s 1}(x)= \begin{cases}u_{01}(x)+k_{11} \hat{v}_{1}(x)+k_{12} \hat{v}_{2}(x), & x \in[0,1], \\
u_{01}(x)+k_{13} \hat{v}_{3}^{*}(x)+k_{14} \hat{v}_{4}(x), & x \in[1,2],\end{cases} \\
& u_{a s 2}(x)= \begin{cases}u_{02}(x)+k_{21} \hat{w}_{1}(x)+k_{22} \hat{w}_{2}(x), & x \in[0,1], \\
u_{02}(x)+k_{23} \hat{w}_{3}^{*}(x)+k_{24} \hat{w}_{4}(x), & x \in[1,2],\end{cases} \\
& \hat{v}_{3}^{*}(x)=\left\{\begin{array}{l}
0, x \in[0,1), \\
\hat{v}_{3}(x), x \in[1,2],
\end{array} \hat{w}_{3}^{*}(x)=\left\{\begin{array}{l}
0, x \in[0,1), \\
\hat{w}_{3}(x), x \in[1,2],
\end{array}\right.\right.
\end{aligned}
$$

where $\bar{u}_{0}(x)=\left(u_{01}(x), u_{02}(x)\right), \hat{v}_{1}(x)=\left[a_{1}(x)\right]^{-\frac{1}{4}} v_{1}(x), \hat{v}_{2}(x)=\left[a_{1}(x)\right]^{-\frac{1}{4}} v_{2}(x)$, $\hat{v}_{3}(x)=\left[a_{1}(x)\right]^{-\frac{1}{4}} v_{3}(x), \hat{v}_{4}(x)=\left[a_{1}(x)\right]^{-\frac{1}{4}} v_{4}(x), \hat{w}_{1}(x)=\left[a_{2}(x)\right]^{-\frac{1}{4}} w_{1}(x), \hat{w}_{2}(x)=$ $\left[a_{2}(x)\right]^{-\frac{1}{4}} w_{2}(x), \hat{w}_{3}(x)=\left[a_{2}(x)\right]^{-\frac{1}{4}} w_{3}(x)$ and $\hat{w}_{4}(x)=\left[a_{2}(x)\right]^{-\frac{1}{4}} w_{4}(x)$.

The constants $k_{i j}, i=1,2, j=1(1) 4$ are to be determined such that $u_{a s 1}, u_{a s 2} \in Y$, $u_{a s 1}(0)=\phi_{1}(0), u_{a s 2}(0)=\phi_{2}(0), u_{a s 1}(2)=l_{1}$ and $u_{a s 2}(2)=l_{2}$. In fact the constants are given by

$$
\left\{\begin{array}{l}
k_{11}=\left\{\left[\phi_{1}(0)-u_{01}(0)\right]-k_{12} \hat{v}_{2}(0)\right\}\left(a_{1}(0)\right)^{1 / 4}, k_{12}=\frac{B_{1}+k_{13} A_{12}}{A_{11}} \\
k_{13}=\frac{B_{2} A_{11}-B_{1} A_{21}}{A_{11} A_{22}+A_{12} A_{21}}, k_{14}=\left\{\left[l_{1}-u_{01}(2)\right]-k_{13} \hat{v}_{3}(2)\right\}\left(a_{1}(2)\right)^{1 / 4} \\
k_{21}=\left\{\left[\phi_{2}(0)-u_{02}(0)\right]-k_{22} \hat{w}_{2}(0)\right\}\left(a_{2}(0)\right)^{1 / 4}, k_{22}=\frac{D_{1}+k_{23} C_{12}}{C_{11}} \\
k_{23}=\frac{D_{2} C_{11}-D_{1} C_{21}}{C_{11} C_{22}+C_{12} C_{21}}, k_{24}=\left\{\left[l_{2}-u_{02}(2)\right]-k_{23} \hat{w}_{3}(2)\right\}\left(a_{2}(2)\right)^{1 / 4}
\end{array}\right.
$$


where

$$
\begin{aligned}
& A_{11}=\left[\left(a_{1}(1)\right)^{-1 / 4}-\left(a_{1}(0)\right)^{1 / 4} \hat{v}_{1}(1) \hat{v}_{2}(0)\right], \\
& A_{12}=\left[\left(a_{1}(1)\right)^{-1 / 4}-\left(a_{1}(2)\right)^{1 / 4} \hat{v}_{3}(2) \hat{v}_{4}(1)\right], \\
& A_{21}=\left(a_{1}(1)\right)^{1 / 4}-\sqrt{\varepsilon}\left(a_{1}(1)\right)^{-5 / 4} a_{1}^{\prime}(1) / 4 \\
& +\hat{v}_{2}(0) \hat{v}_{1}(1)\left(a_{1}(0)\right)^{1 / 4}\left[\sqrt{\varepsilon}\left(a_{1}(1)\right)^{-1} a_{1}^{\prime}(1) / 4+\sqrt{a_{1}}(1)\right], \\
& A_{22}=\left(a_{1}(1)\right)^{1 / 4}+\sqrt{\varepsilon}\left(a_{1}(1)\right)^{-5 / 4} a_{1}^{\prime}(1) / 4 \\
& +\hat{v}_{3}(2) \hat{v}_{4}(1)\left(a_{1}(2)\right)^{1 / 4}\left[-\sqrt{\varepsilon}\left(a_{1}(1)\right)^{-1} a_{1}^{\prime}(1) / 4+\sqrt{a_{1}}(1)\right], \\
& B_{1}=\left[u_{01}(1+)-u_{01}(1-)\right]+\left[\left(l_{1}-u_{01}(2)\right)\left(a_{1}(2)\right)^{1 / 4} \hat{v}_{4}(1)\right] \\
& +\left[\left(u_{01}(0)-\phi_{1}(0)\right)\left(a_{1}(0)\right)^{1 / 4} \hat{v}_{1}(1)\right], \\
& B_{2}=\sqrt{\varepsilon}\left[u_{01}^{\prime}(1+)-u_{01}^{\prime}(1-)\right]+\left[\phi_{1}(0)-u_{01}(0)\right] \hat{v}_{1}(1)\left(a_{1}(0)\right)^{1 / 4}\left[\sqrt{a_{1}(1)}+\sqrt{\varepsilon} \frac{a_{1}^{\prime}(1)}{4 a_{1}(1)}\right] \\
& +\hat{v}_{4}(1)\left(a_{1}(2)\right)^{1 / 4}\left[l_{1}-u_{01}(2)\right]\left[\sqrt{a_{1}(1)}-\sqrt{\varepsilon} \frac{a_{1}^{\prime}(1)}{4 a_{1}(1)}\right] \\
& C_{11}=\left[\left(a_{2}(1)\right)^{-1 / 4}-\left(a_{2}(0)\right)^{1 / 4} \hat{w}_{1}(1) \hat{w}_{2}(0)\right], \\
& C_{12}=\left[\left(a_{2}(1)\right)^{-1 / 4}-\left(a_{2}(2)\right)^{1 / 4} \hat{w}_{3}(2) \hat{w}_{4}(1)\right], \\
& C_{21}=\left(a_{2}(1)\right)^{1 / 4}-\sqrt{\varepsilon}\left(a_{2}(1)\right)^{-5 / 4} a_{2}^{\prime}(1) / 4 \\
& +\hat{w}_{2}(0) \hat{w}_{1}(1)\left(a_{2}(0)\right)^{1 / 4}\left[\sqrt{\varepsilon}\left(a_{2}(1)\right)^{-1} a_{2}^{\prime}(1) / 4+\sqrt{a_{2}}(1)\right], \\
& C_{22}=\left(a_{2}(1)\right)^{1 / 4}+\sqrt{\varepsilon}\left(a_{2}(1)\right)^{-5 / 4} a_{2}^{\prime}(1) / 4 \\
& +\hat{w}_{3}(2) \hat{w}_{4}(1)\left(a_{2}(2)\right)^{1 / 4}\left[-\sqrt{\varepsilon}\left(a_{2}(1)\right)^{-1} a_{2}^{\prime}(1) / 4+\sqrt{a_{2}}(1)\right], \\
& D_{1}=\left[u_{02}(1+)-u_{02}(1-)\right]+\left[\left(l_{2}-u_{02}(2)\right)\left(a_{2}(2)\right)^{1 / 4} \hat{w}_{4}(1)\right] \\
& +\left[\left(u_{02}(0)-\phi_{2}(0)\right)\left(a_{2}(0)\right)^{1 / 4} \hat{w}_{1}(1)\right], \\
& D_{2}=\sqrt{\varepsilon}\left[u_{02}^{\prime}(1+)-u_{02}^{\prime}(1-)\right]+\left[\phi_{2}(0)-u_{02}(0)\right] \hat{w}_{1}(1)\left(a_{2}(0)\right)^{1 / 4}\left[\sqrt{a_{2}(1)}+\sqrt{\varepsilon} \frac{a_{2}^{\prime}(1)}{4 a_{2}(1)}\right] \\
& +\hat{w}_{4}(1)\left(a_{2}(2)\right)^{1 / 4}\left[l_{2}-u_{02}(2)\right]\left[\sqrt{a_{2}(1)}-\sqrt{\varepsilon} \frac{a_{2}^{\prime}(1)}{4 a_{2}(1)}\right] .
\end{aligned}
$$

It is easy to see that $\left|k_{i j}\right| \leq C, i=1,2, j=1(1) 4$.

Theorem 4.1. Let $\bar{u}=\left(u_{1}, u_{2}\right)$ be the solution of $(2.1)$ and $\bar{u}_{a s}=\left(u_{a s 1}, u_{a s 2}\right)$ be its asymptotic expansion approximation given by (4.10) and (4.11). Then,

$$
\left\|u_{i}-u_{a s i}\right\|_{\bar{\Omega}} \leq C \varepsilon, i=1,2 .
$$


Proof. Consider the barrier function $\bar{\varphi}^{ \pm}=\left(\varphi_{1}^{ \pm}, \varphi_{2}^{ \pm}\right)$, where

$$
\begin{gathered}
\varphi_{i}^{ \pm}(x)=\left\{\begin{array}{r}
C_{1} \varepsilon\left[s_{i}(x)+e^{-\sqrt{\frac{\alpha}{\varepsilon}} x}+e^{-\sqrt{\frac{\alpha}{\varepsilon}}(1-x)}+1+e^{-\sqrt{\frac{\alpha}{\varepsilon}}(2-x)}\right] \\
\pm\left(u_{i}(x)-u_{\text {asi }}(x)\right), x \in[0,1], \\
C_{1} \varepsilon\left[s_{i}(x)+e^{-\sqrt{\frac{\alpha}{\varepsilon}} x}+1+\tilde{v}_{3}^{*}(x)+e^{-\sqrt{\frac{\alpha}{\varepsilon}}(2-x)}\right] \\
\pm\left(u_{i}(x)-u_{\text {asi }}(x)\right), x \in[1,2], i=1,2
\end{array}\right. \\
\tilde{v}_{3}^{*}(x)=\tilde{w}_{3}^{*}(x)=\left\{\begin{array}{c}
0, x \in[0,1), \\
e^{-\sqrt{\frac{\alpha}{\varepsilon}}(x-1)}, x \in[1,2],
\end{array}\right.
\end{gathered}
$$

and $C_{1}>0$ is a constant independent of $\varepsilon$. It is easy to see that $\varphi_{i}^{ \pm} \in C^{0}(\bar{\Omega}) \cap C^{2}\left(\Omega^{*}\right)$, $\varphi_{i}^{ \pm}(0)>0$ and $\varphi_{i}^{ \pm}(2)>0, i=1,2$. Let $x \in \Omega^{-}$. First we have,

$$
\begin{aligned}
P_{1}\left(\bar{u}(x)-\bar{u}_{a s}(x)\right) & =\varepsilon\left[u_{01}^{\prime \prime}(x)+k_{11} \hat{v}_{1}(x)\left[\frac{5\left(a_{1}^{\prime}(x)\right)^{2}}{16\left(a_{1}(x)\right)^{2}}-\frac{a_{1}^{\prime \prime}(x)}{4 a_{1}(x)}\right]\right] \\
& +\varepsilon\left[k_{12} \hat{v}_{2}(x)\left[\frac{5\left(a_{1}^{\prime}(x)\right)^{2}}{16\left(a_{1}(x)\right)^{2}}-\frac{a_{1}^{\prime \prime}(x)}{4 a_{1}(x)}\right]\right] \\
& \geq-C \varepsilon
\end{aligned}
$$

since $\left|k_{11}\right|,\left|k_{12}\right| \leq C,\left\|u_{01}^{(2)}\right\|_{\Omega^{*}} \leq C$, and $a_{1}$ is sufficiently smooth on $\bar{\Omega}$. Then,

$$
\begin{aligned}
& P_{1} \bar{\varphi}^{ \pm}(x)=C_{1} \varepsilon\left[\left[a_{1}(x)-\alpha\right] e^{-\sqrt{\frac{\alpha}{\varepsilon}} x}+\left[a_{1}(x)-\alpha\right] e^{-\sqrt{\frac{\alpha}{\varepsilon}}(1-x)}\right. \\
& \left.+\left[a_{1}(x)-\alpha\right] e^{-\sqrt{\frac{\alpha}{\varepsilon}}(2-x)}\right]+C_{1} \varepsilon\left[a_{1}(x)\left[S_{1}(x)+1\right]\right] \pm P_{1}\left(\bar{u}(x)-\bar{u}_{a s}(x)\right) \\
& \quad \geq C_{1} \varepsilon\left[\left[\alpha_{1}-\alpha\right] e^{-\sqrt{\frac{\alpha}{\varepsilon}} x}+\left[\alpha_{1}-\alpha\right] e^{-\sqrt{\frac{\alpha}{\varepsilon}}(1-x)}+\left[\alpha_{1}-\alpha\right] e^{-\sqrt{\frac{\alpha}{\varepsilon}}(2-x)}\right] \\
& +C_{1} \varepsilon \frac{9 \alpha_{1}}{8} \mp C \varepsilon\left[1+e^{-\sqrt{\frac{\alpha}{\varepsilon}} x}+e^{-\sqrt{\frac{\alpha}{\varepsilon}}(1-x)}\right] .
\end{aligned}
$$

Hence $P_{1} \bar{\varphi}^{ \pm}(x) \geq 0$ for a suitable choice of $C_{1}$. Also it is easy to see that $P_{2} \bar{\varphi}^{ \pm}(x) \geq 0$ for a suitable choice of $C_{1}$.

Similarly one can show that $P_{i} \bar{\varphi}^{ \pm}(x) \geq 0, x \in \Omega^{+}, i=1,2$. Further, we have $\left[\varphi_{i}^{ \pm^{\prime}}\right](1)<$ $0, i=1,2$. Then by the Theorem 3.1 , we have $\varphi_{i}^{ \pm}(x) \geq 0, x \in \bar{\Omega}$, that is,

$$
\left|u_{i}(x)-u_{a s i}(x)\right| \leq C \varepsilon, x \in \bar{\Omega}, i=1,2 \text {. }
$$

\section{Numerical Methods}

In this section, hybrid finite difference schemes for the first order singularly perturbed problems (4.2)-(4.9) are described. 
5.1. Mesh Selection Strategy. The BVP (2.1) exhibits strong boundary layers at $x=0, x=$ 2 and strong interior layers (left and right) at $x=1$. Therefore, we choose a piece-wise uniform Shishkin mesh on $\bar{\Omega}$. For this we divide the interval $[0,2]$ in to six subintervals, namely $\Omega_{1}=$ $[0, \sigma], \Omega_{2}=[\sigma, 1-\sigma], \Omega_{3}=[1-\sigma, 1], \Omega_{4}=[1,1+\sigma], \Omega_{5}=[1+\sigma, 2-\sigma]$ and $\Omega_{6}=[2-\sigma, 2]$ where, $\sigma=\min \left\{0.25, \frac{2 \sqrt{\varepsilon} \log N}{\sqrt{\alpha}}\right\}$. Let $h=4 N^{-1} \tau$ and $H=2 N^{-1}(1-2 \tau)$. The mesh $\bar{\Omega}^{2 N}=\left\{x_{0}, x_{1}, \ldots, x_{2 N}\right\}$ is defined by

$$
\begin{gathered}
x_{0}=0.0, x_{i}=x_{0}+i h, i=1(1) \frac{N}{4}, x_{i+\frac{N}{4}}=x_{\frac{N}{4}}+i H, i=1(1) \frac{N}{2}, \\
x_{i+\frac{3 N}{4}}=x_{\frac{3 N}{4}}+i h, i=1(1) \frac{N}{4}, x_{i+N}=x_{N}+i h, i=1(1) \frac{N}{4}, \\
x_{i+\frac{5 N}{4}}=x_{\frac{5 N}{4}}+i H, i=1(1) \frac{N}{2}, x_{i+\frac{7 N}{4}}=x_{\frac{7 N}{4}}+i h, i=1(1) \frac{N}{4} .
\end{gathered}
$$

5.2. Hybrid Finite Difference Schemes for the Problems (4.6) - (4.9). Applying the hybrid finite difference scheme given in $[22,23]$ to the above singularly perturbed problems $(4.6)$ (4.9), we get

$$
\begin{aligned}
& L_{1}^{N} V_{1_{i}}=\left\{\begin{array}{l}
\sqrt{\varepsilon} \frac{V_{1_{i}}-V_{1_{i-1}}}{h}+\sqrt{a_{1_{i-1}}} \frac{V_{1_{i}}+V_{1_{i-1}}}{2}=0, i=1(1) \frac{N}{4}, \\
\sqrt{\varepsilon} \frac{V_{1_{i}}-V_{1_{i-1}}}{H}+\sqrt{a_{1_{i}}} V_{1_{i}}=0 i=\frac{N}{4}+1(1) \frac{3 N}{4}, \\
\sqrt{\varepsilon} \frac{V_{1_{i}}-V_{1_{i-1}}}{h}+\sqrt{a_{1_{i}}} V_{1_{i}}=0, i=\frac{3 N}{4}+1(1) N, \\
\sqrt{\varepsilon} \frac{V_{1_{i}}-V_{1_{i-1}}}{h}+\sqrt{a_{1_{i}}} V_{1_{i}}=0, i=N+1(1) \frac{5 N}{4}, \\
\sqrt{\varepsilon} \frac{V_{1_{i}}-V_{1_{i-1}}}{H}+\sqrt{a_{1_{i}}} V_{1_{i}}=0, i=\frac{5 N}{4}+1(1) \frac{7 N}{4}, \\
\sqrt{\varepsilon} \frac{V_{1_{i}}-V_{1_{i-1}}}{h}+\sqrt{a_{1_{i}}} V_{1_{i}}=0, i=\frac{7 N}{4}+1(1) 2 N,
\end{array}\right. \\
& V_{1_{0}}=1 \text {, } \\
& L_{2}^{N} V_{2_{i}}=\left\{\begin{array}{l}
\sqrt{\varepsilon} \frac{V_{2_{i+1}}-V_{2_{i}}}{h}-\sqrt{a_{1_{i}}} V_{2_{i}}=0, i=0(1) \frac{N}{4}, \\
\sqrt{\varepsilon} \frac{V_{2_{i+1}}-V_{2_{i}}}{H}-\sqrt{a_{1_{i}}} V_{2_{i}}=0, i=\frac{N}{4}+1(1) \frac{3 N}{4}, \\
\sqrt{\varepsilon} \frac{V_{2_{i+1}}-V_{2_{i}}}{h}-\sqrt{a_{1_{i+1} / 2}} \frac{V_{2_{i+1}}+V_{2_{i}}}{2}=0, i=\frac{3 N}{4}+1(1) N-1,
\end{array}\right. \\
& V_{2_{N}}=1 \text {, } \\
& \begin{array}{l}
L_{1}^{N} V_{3_{i}}=\left\{\begin{array}{l}
\sqrt{\varepsilon} \frac{V_{3_{i}}-V_{3_{i-1}}}{h}+\sqrt{a_{1_{i-1 / 2}}} \frac{V_{3_{i-1}}+V_{3_{i}}}{2}=0, i=N+1(1) \frac{5 N}{4}, \\
\sqrt{\varepsilon} \frac{V_{3_{i}}-V_{3_{i-1}}}{H}+\sqrt{a_{1_{i}}} V_{3_{i}}=0, i=\frac{5 N}{4}+1(1) \frac{7 N}{4} \\
\sqrt{\varepsilon} \frac{V_{3_{i}}-V_{3_{i-1}}}{h}+\sqrt{a_{1_{i}}} V_{3_{i}}=0, i=\frac{7 N}{4}+1(1) 2 N
\end{array}\right. \\
V_{3_{N}}=1
\end{array}
\end{aligned}
$$




$$
L_{3}^{N} V_{4_{i}}=\left\{\begin{array}{l}
\sqrt{\varepsilon} \frac{V_{4_{i+1}}-V_{4_{i}}}{h}-\sqrt{a_{1_{i}}} V_{4_{i}}=0, i=0(1) \frac{N}{4}, \\
\sqrt{\varepsilon} \frac{V_{4_{i+1}}-V_{4_{i}}}{H}-\sqrt{a_{1_{i}}} V_{4_{i}}=0, i=\frac{N}{4}+1(1) \frac{3 N}{4}, \\
\sqrt{\varepsilon} \frac{V_{4_{i+1}}-V_{4_{i}}}{h}-\sqrt{a_{1_{i}}} V_{4_{i}}=0, i=\frac{3 N}{4}+1(1) N \\
\sqrt{\varepsilon} \frac{V_{4_{i+1}}-V_{4_{i}}}{h}-\sqrt{a_{1}} V_{4_{i}}=0, i=N+1(1) \frac{5 N}{4}, \\
\sqrt{\varepsilon} \frac{V_{4_{i+1}}-V_{4_{i}}}{H}-\sqrt{a_{1_{i}}} V_{4_{i}}=0, i=\frac{5 N}{4}+1(1) \frac{7 N}{4}, \\
\sqrt{\varepsilon} \frac{V_{4_{i+1}}-V_{4_{i}}}{h}-\sqrt{a_{1_{i+1} / 2}} \frac{V_{4_{i+1}}+V_{4_{i}}}{2}=0, i=\frac{7 N}{4}+1(1) 2 N-1,
\end{array}\right.
$$

$V_{4_{2 N}}=1$,

$$
L_{4}^{N} W_{1_{i}}=\left\{\begin{array}{l}
\sqrt{\varepsilon} \frac{W_{1_{i}}-W_{1_{i-1}}}{h}+\sqrt{a_{2_{i-1}}} \frac{W_{1_{i}}+W_{1_{i-1}}}{2}=0, i=1(1) \frac{N}{4}, \\
\sqrt{\varepsilon} \frac{W_{1_{i}}-W_{1_{i-1}}}{H}+\sqrt{a_{2_{i}}} W_{1_{i}},=0 i=\frac{N}{4}+1(1) \frac{3 N}{4}, \\
\sqrt{\varepsilon} \frac{W_{1_{i}}-W_{1_{i-1}}}{h}+\sqrt{a_{2_{i}}} W_{1_{i}}=0, i=\frac{3 N}{4}+1(1) N, \\
\sqrt{\varepsilon} \frac{W_{1_{i}}-W_{1_{i-1}}}{h}+\sqrt{a_{2_{i}}} W_{1_{i}}=0, i=N+1(1) \frac{5 N}{4}, \\
\sqrt{\varepsilon} \frac{W_{1_{i}}-W_{1_{i-1}}}{H}+\sqrt{a_{2_{i}}} W_{1_{i}}=0, i=\frac{5 N}{4}+1(1) \frac{7 N}{4}, \\
\sqrt{\varepsilon} \frac{W_{1_{i}}-W_{1_{i-1}}}{h}+\sqrt{a_{2_{i}}} W_{1_{i}}=0, i=\frac{7 N}{4}+1(1) 2 N,
\end{array}\right.
$$

$W_{1_{0}}=1$,

$$
L_{5}^{N} W_{2_{i}}=\left\{\begin{array}{l}
\sqrt{\varepsilon} \frac{W_{2_{i+1}}-W_{2_{i}}}{h}-\sqrt{a_{2_{i}}} W_{2_{i}}=0, i=0(1) \frac{N}{4}, \\
\sqrt{\varepsilon} \frac{W_{2_{i+1}}-W_{2_{i}}}{H}-\sqrt{a_{2_{i}}} W_{2_{i}}=0, i=\frac{N}{4}+1(1) \frac{3 N}{4}, \\
\sqrt{\varepsilon} \frac{W_{2_{i+1}}-W_{2_{i}}}{h}-\sqrt{a_{2_{i+1 / 2}}} \frac{W_{2_{i+1}}+W_{2_{i}}}{2}=0, i=\frac{3 N}{4}+1(1) N-1,
\end{array}\right.
$$

$W_{2_{N}}=1$,

$$
L_{4}^{N} W_{3_{i}}=\left\{\begin{array}{l}
\sqrt{\varepsilon} \frac{W_{3_{i}}-W_{3_{i-1}}}{h}+\sqrt{a_{2_{i-1 / 2}}} \frac{W_{3_{i-1}}+W_{3_{i}}}{2}=0, i=N+1(1) \frac{5 N}{4}, \\
\sqrt{\varepsilon} \frac{W_{3_{i}}-W_{3_{i-1}}}{H}+\sqrt{a_{2_{i}}} W_{3_{i}}=0, i=\frac{5 N}{4}+1(1) \frac{7 N}{4} \\
\sqrt{\varepsilon} \frac{W_{3_{i}}-W_{3_{i-1}}}{h}+\sqrt{a_{2_{i}}} W_{3_{i}}=0, i=\frac{7 N}{4}+1(1) 2 N
\end{array}\right.
$$

$W_{3_{N}}=1$, 
and

$$
L_{6}^{N} W_{4_{i}}=\left\{\begin{array}{l}
\sqrt{\varepsilon} \frac{W_{4_{i+1}}-W_{4_{i}}}{h}-\sqrt{a_{2_{i}}} W_{4_{i}}=0, i=0(1) \frac{N}{4}, \\
\sqrt{\varepsilon} \frac{W_{4_{i+1}}-W_{4_{i}}}{H}-\sqrt{a_{2_{i}}} W_{4_{i}}=0, i=\frac{N}{4}+1(1) \frac{3 N}{4}, \\
\sqrt{\varepsilon} \frac{W_{4_{i+1}}-W_{4_{i}}}{h}-\sqrt{a_{2_{i}}} W_{4_{i}}=0, i=\frac{3 N}{4}+1(1) N, \\
\sqrt{\varepsilon} \frac{W_{4_{i+1}}-W_{4_{i}}}{h}-\sqrt{a_{2_{i}}} W_{4_{i}}=0, i=N+1(1) \frac{5 N}{4}, \\
\sqrt{\varepsilon} \frac{W_{4_{i+1}}-W_{4_{i}}}{H}-\sqrt{a_{2_{i}}} W_{4_{i}}=0, i=\frac{5 N}{4}+1(1) \frac{7 N}{4}, \\
\sqrt{\varepsilon} \frac{W_{4_{i+1}}-W_{4_{i}}}{h}-\sqrt{a_{a_{i+1}}} \frac{W_{4_{i+1}}+W_{4_{i}}}{2}=0, i=\frac{7 N}{4}+1(1) 2 N-1,
\end{array}\right.
$$

$W_{4_{2 N}}=1$,

where $a_{1_{i}}=a_{1}\left(x_{i}\right)$ and $a_{1_{i+1 / 2}}=a_{1}\left(\frac{x_{i}+x_{i+1}}{2}\right)$, the similar expressions are true for other functions. The following theorem gives an error estimate for this scheme.

Theorem 5.1. Let $v_{1}(x), v_{2}(x), v_{3}(x), v_{4}(x), w_{1}(x), w_{2}(x), w_{3}(x)$ and $w_{4}(x)$ be the solutions of the problems (4.2)-(4.9), respectively. Further let $V_{1}=\left(V_{1_{0}}, \cdots, V_{1_{2 N}}\right), V_{2}=$ $\left(V_{2_{0}}, \cdots, V_{2_{N}}\right), V_{3}=\left(V_{3_{N}}, \cdots, V_{3_{2 N}}\right), V_{4}=\left(V_{4_{0}}, \cdots, V_{4_{2 N}}\right), W_{1}=\left(W_{1_{0}}, \cdots, W_{1_{2 N}}\right)$, $W_{2}=\left(W_{2_{0}}, \cdots, W_{2_{N}}\right), W_{3}=\left(W_{3_{N}}, \cdots, W_{3_{2 N}}\right)$ and $W_{4}=\left(W_{4_{0}}, \cdots, W_{4_{2 N}}\right)$ be their numerical solutions defined by (5.1)-(5.8). Then

$$
\begin{aligned}
& \left\|v_{1}-V_{1}\right\|_{\bar{\Omega}^{2 N}} \leq C N^{-2} \log ^{2} N,\left\|v_{2}-V_{2}\right\|_{\tilde{\Omega}^{-}} \leq C N^{-2} \log ^{2} N, \\
& \left\|v_{3}-V_{3}\right\|_{\Omega^{+}} \leq C N^{-2} \log ^{2} N,\left\|v_{4}-V_{4}\right\|_{\bar{\Omega}^{2 N}} \leq C N^{-2} \log ^{2} N, \\
& \left\|w_{1}-W_{1}\right\|_{\bar{\Omega}^{2 N}} \leq C N^{-2} \log ^{2} N,\left\|w_{2}-W_{2}\right\|_{\Omega^{-}} \leq C N^{-2} \log ^{2} N, \\
& \left\|w_{3}-W_{3}\right\|_{\Omega^{+}} \leq C N^{-2} \log ^{2} N,\left\|w_{4}-W_{4}\right\|_{\bar{\Omega}^{2 N}} \leq C N^{-2} \log ^{2} N,
\end{aligned}
$$

where $\tilde{\Omega^{-}}=\bar{\Omega}^{2 N} \cap[0,1]$ and $\tilde{\Omega^{+}}=\bar{\Omega}^{2 N} \cap[1,2]$.

Proof. See [22].

Let $\bar{U}_{0_{i}}=\left(U_{01_{i}}, U_{02_{i}}\right)$ be the values of the solution of the problem (4.1) given by

$$
\begin{gathered}
U_{01_{i}}=\left\{\begin{array}{l}
\frac{f_{1}\left(x_{i}\right)}{a_{1}\left(x_{i}\right)}-\frac{b_{11}\left(x_{i}\right)}{a_{1}\left(x_{i}\right)} \phi_{1}\left(x_{i}-1\right)-\frac{b_{12}\left(x_{i}\right)}{a_{1}\left(x_{i}\right)} \phi_{2}\left(x_{i}-1\right), i=0(1) N, \\
\frac{f_{1}\left(x_{i}\right)}{a_{1}\left(x_{i}\right)}-\frac{b_{11}\left(x_{i}\right)}{a_{1}\left(x_{i}\right)} U_{01_{i-N}}-\frac{b_{12}\left(x_{i}\right)}{a_{1}\left(x_{i}\right)} U_{02_{i-N}}, i=N+1(1) 2 N,
\end{array}\right. \\
U_{02_{i}}=\left\{\begin{array}{l}
\frac{f_{2}\left(x_{i}\right)}{a_{2}\left(x_{i}\right)}-\frac{b_{21}\left(x_{i}\right)}{a_{2}\left(x_{i}\right)} \phi_{1}\left(x_{i}-1\right)-\frac{b_{22}\left(x_{i}\right)}{a_{2}\left(x_{i}\right)} \phi_{2}\left(x_{i}-1\right), i=0(1) N, \\
\frac{f_{2}\left(x_{i}\right)}{a_{2}\left(x_{i}\right)}-\frac{b_{21}\left(x_{i}\right)}{a_{2}\left(x_{i}\right)} U_{01_{i-N}}-\frac{b_{22}\left(x_{i}\right)}{a_{2}\left(x_{i}\right)} U_{02_{i-N}}, i=N+1(1) 2 N .
\end{array}\right.
\end{gathered}
$$

5.3. A Numerical Solution to the BVP (2.1). A numerical solution $\bar{U}_{i}=\left(U_{1_{i}}, U_{2_{i}}\right)$ of the original problem (2.1) is given by

$$
U_{1_{i}}=\left\{\begin{array}{l}
U_{01_{i}}+k_{11}\left[a_{1_{i}}\right]^{-1 / 4} V_{1_{i}}+k_{12}\left[a_{1_{i}}\right]^{-1 / 4} V_{2_{i}}, i=0(1) N \\
U_{01_{i}}+k_{13}\left[a_{1_{i}}\right]^{-1 / 4} V_{3_{i}}+k_{14}\left[a_{1_{i}}\right]^{-1 / 4} V_{4_{i}}, i=N+1(1) 2 N
\end{array}\right.
$$




$$
U_{2_{i}}=\left\{\begin{array}{l}
U_{02_{i}}+k_{21}\left[a_{2_{i}}\right]^{-1 / 4} W_{1_{i}}+k_{22}\left[a_{2_{i}}\right]^{-1 / 4} W_{2_{i}}, i=0(1) N, \\
U_{02_{i}}+k_{23}\left[a_{2_{i}}\right]^{-1 / 4} W_{3_{i}}+k_{24}\left[a_{2_{i}}\right]^{-1 / 4} W_{4_{i}}, i=N+1(1) 2 N,
\end{array}\right.
$$

where $\bar{U}_{0_{i}}, V_{1_{i}}, V_{2_{i}}, V_{3_{i}}, V_{4_{i}}, W_{1_{i}}, W_{2_{i}}, W_{3_{i}}$ and $W_{4_{i}}$ are numerical solutions of the problems (4.1)- (4.9), respectively and $k_{i j}, i=1,2, j=1(1) 4$ are defined by (4.12). An error estimate for this numerical solution is derived in the following section.

\section{AN ERROR ESTIMATE}

Theorem 6.1. Let $\bar{u}(x)$ be the solution of the problem (2.1). Further let $\bar{U}_{i}=\left(U_{1_{i}}, U_{2_{i}}\right)$ be its numerical solution defined by (5.11) and (5.12). Then

$$
\left|u_{k}\left(x_{i}\right)-U_{k_{i}}\right| \leq C\left(N^{-2} \log ^{2} N\right), i=0(1) 2 N, k=1,2 .
$$

Proof. From Theorems 4.1 and 5.1 we have

$$
\begin{aligned}
& \left\|u_{i}(x)-u_{a s i}(x)\right\|_{\bar{\Omega}} \leq C \varepsilon, i=1,2,\left\|v_{1}-V_{1}\right\|_{\bar{\Omega}^{2 N}} \leq C N^{-2} \log ^{2} N, \\
& \left\|v_{2}-V_{2}\right\|_{\Omega^{-} \cap \bar{\Omega}^{2 N}} \leq C N^{-2} \log ^{2} N,\left\|v_{3}-V_{3}\right\|_{\Omega^{+} \cap \bar{\Omega}^{2 N}} \leq C N^{-2} \log ^{2} N, \\
& \left\|v_{4}-V_{4}\right\|_{\bar{\Omega}^{2 N}} \leq C N^{-2} \log ^{2} N,\left\|w_{1}-W_{1}\right\|_{\bar{\Omega}^{2 N}} \leq C N^{-2} \log ^{2} N, \\
& \left\|w_{2}-W_{2}\right\|_{\Omega^{-} \cap \bar{\Omega}^{2 N}} \leq C N^{-2} \log ^{2} N,\left\|w_{3}-W_{3}\right\|_{\Omega^{+} \cap \bar{\Omega}^{2 N}} \leq C N^{-2} \log ^{2} N, \\
& \left\|w_{4}-W_{4}\right\|_{\bar{\Omega}^{2 N}} \leq C N^{-2} \log ^{2} N .
\end{aligned}
$$

Then

$$
\begin{aligned}
& \left|u_{1}\left(x_{i}\right)-U_{1_{i}}\right| \leq\left|u_{1}\left(x_{i}\right)-u_{a s 1}\left(x_{i}\right)\right|+\left|u_{a s 1}\left(x_{i}\right)-U_{1_{i}}\right|, i=0(1) 2 N \\
\leq & \left\{\begin{array}{c}
\left|u_{1}\left(x_{i}\right)-u_{a s 1}\left(x_{i}\right)\right|+\left|u_{01}\left(x_{i}\right)-U_{01_{i}}\right|+\left|k_{11}\right|\left|a_{1_{i}}^{-1 / 4}\right|\left|v_{1}\left(x_{i}\right)-V_{1_{i}}\right| \\
+\left|k_{12}\right|\left|a_{1_{i}}^{-1 / 4}\right|\left|v_{2}\left(x_{i}\right)-V_{2_{i}}\right|, i=0(1) N \\
\left|u_{1}\left(x_{i}\right)-u_{a s 1}\left(x_{i}\right)\right|+\left|u_{01}\left(x_{i}\right)-U_{01_{i}}\right|+\left|k_{13}\right|\left|a_{1_{i}}^{-1 / 4}\right|\left|v_{3}\left(x_{i}\right)-V_{3_{i}}\right| \\
+\left|k_{14}\right|\left|a_{1_{i}}^{-1 / 4}\right|\left|v_{4}\left(x_{i}\right)-V_{4_{i}}\right|, i=N+1(1) 2 N
\end{array}\right. \\
\leq & C \varepsilon+C N^{-2} \log ^{2} N, i=0(1) 2 N .
\end{aligned}
$$

That is,

$$
\left|u_{1}\left(x_{i}\right)-U_{1_{i}}\right| \leq C\left(\varepsilon+N^{-2} \log ^{2} N\right), i=0(1) 2 N .
$$

It is easy to see that

$$
\left|u_{2}\left(x_{i}\right)-U_{2_{i}}\right| \leq C\left(\varepsilon+N^{-2} \log ^{2} N\right), i=0(1) 2 N .
$$

Since it is assumed that $\sqrt{\varepsilon} \leq C N^{-1}$, we have

$$
\left|u_{k}\left(x_{i}\right)-U_{k_{i}}\right| \leq C\left(N^{-2} \log ^{2} N\right), i=0(1) 2 N, k=1,2 .
$$




\section{Discontinuous Source Term}

In the previous section it was assumed that $f_{k}, k=1,2$ are smooth on $[0,2]$. Motivated by the works of $[8,9,24]$ we suppose that $f_{1}(x)$ and $f_{2}(x)$ have a simple discontinuity at $x=1$, that is, $f_{1}(1-) \neq f_{1}(1+)$ and $f_{2}(1-) \neq f_{2}(1+)$. Consider the following BVP with discontinuous source term.

Find $\bar{u}(x)=\left(u_{1}(x), u_{2}(x)\right), u_{1}, u_{2} \in Y$ such that

$$
\left\{\begin{array}{l}
-\varepsilon u_{1}^{\prime \prime}(x)+a_{1}(x) u_{1}(x)+\sum_{k=1}^{2} b_{1 k} u_{k}(x-1)=f_{1}(x), x \in \Omega^{*}, \\
-\varepsilon u_{2}^{\prime \prime}(x)+a_{2}(x) u_{2}(x)+\sum_{k=1}^{2} b_{2 k} u_{k}(x-1)=f_{2}(x), x \in \Omega^{*}, \\
u_{1}(x)=\phi_{1}(x), x \in[-1,0], u_{1}(2)=l_{1}, \\
u_{2}(x)=\phi_{2}(x), x \in[-1,0], u_{2}(2)=l_{2}, \\
f_{j}(1-) \neq f_{j}(1+), j=1,2,
\end{array}\right.
$$

where it is assumed that the conditions stated in Section 2 for the coefficients and the relations among $a_{i}, b_{i j}, c_{i j}$ and $\phi_{i}, i, j=1,2$ hold true and $f_{i}, i=1,2$ are smooth in $\Omega^{*}$. It can be easily verified that the maximum principle and stability result are valid for the above problem (7.1). Now, an asymptotic expansion for the solution of the problem (7.1) is given by (4.10) and (4.11). We can prove a similar result of Theorem 4.1 for the (7.1). With regard to the numerical method, the same mesh selection strategy described in Section 5.1 can be adopted here.

\section{NumeriCAl Results}

In this section, two examples are given to illustrate the numerical method discussed in this paper. The exact solutions of the test problems are not known. Therefore, we use the double mesh principle to estimate the error and compute the experiment rate of convergence in our computed solution. For this we put

$$
D_{k, \varepsilon}^{M}=\max _{0 \leq i \leq M}\left|U_{k_{i}}^{M}-U_{k_{2 i}}^{2 M}\right|, k=1,2
$$

where $U_{k_{i}}^{M}$ and $U_{k_{2 i}}^{2 M}$ are the $i^{\text {th }}$ and $2 i^{t h}$ components of the numerical solutions on meshes of $M$ and $2 M$ points respectively, here $M=2 N$. We compute the uniform error and rate of convergence as

$$
D_{k}^{M}=\max _{\varepsilon} D_{k, \varepsilon}^{M}, p_{k}^{M}=\log _{2}\left(\frac{D_{k}^{M}}{D_{k}^{2 M}}\right), k=1,2
$$

For the following examples the numerical results are presented for the values of perturbation parameter $\sqrt{\varepsilon}=2^{-11}, 2^{-12}, 2^{-13}, 2^{-14}, 2^{-15}, 2^{-16}, 2^{-17}$. 
Example 8.1. (Continuous Source Term)

$$
\left\{\begin{array}{l}
-\varepsilon u_{1}^{\prime \prime}(x)+11 u_{1}(x)-\left(x^{2}+1\right) u_{1}(x-1)-(x+1) u_{2}(x-1)=\exp (x), x \in \Omega^{*}, \\
-\varepsilon u_{2}^{\prime \prime}(x)+16 u_{2}(x)-x u_{1}(x-1)-x u_{2}(x-1)=\exp (x), x \in \Omega^{*} \\
u_{1}(x)=1, x \in[-1,0], u_{1}(2)=1 \\
u_{2}(x)=1, x \in[-1,0], u_{2}(2)=1 .
\end{array}\right.
$$

Table 1 presents the values of $D_{k}^{M}$ and $p_{k}^{M}, k=1,2$ for this problem.

Example 8.2. (Discontinuous Source Term)

$$
\left\{\begin{array}{l}
-\varepsilon u_{1}^{\prime \prime}(x)+11 u_{1}(x)-\left(x^{2}+1\right) u_{1}(x-1)-(x+1) u_{2}(x-1)=\left\{\begin{array}{l}
-1, x \in \Omega^{-}, \\
1, x \in \Omega^{+},
\end{array}\right. \\
-\varepsilon u_{2}^{\prime \prime}(x)+16 u_{2}(x)-x u_{1}(x-1)-x u_{2}(x-1)=\left\{\begin{array}{l}
1, x \in \Omega^{-}, \\
-1, x \in \Omega^{+},
\end{array}\right. \\
u_{1}(x)=1, x \in[-1,0], u_{1}(2)=1, \\
u_{2}(x)=1, x \in[-1,0], u_{2}(2)=1 .
\end{array}\right.
$$

Table 2 presents the values of $D_{k}^{M}$ and $p_{k}^{M}, k=1,2$ for this problem. Further, Figures 1 and 2 represent the numerical solutions of the above Examples 8.1 and 8.2 respectively.

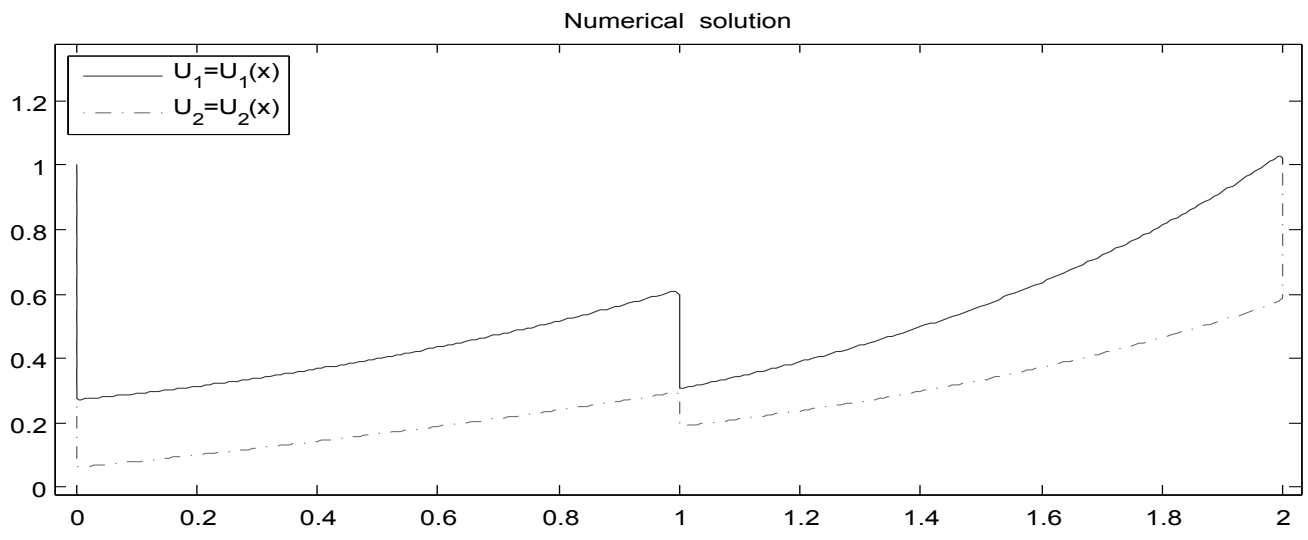

FIGURE 1. Numerical solution of the above Example 8.1. 


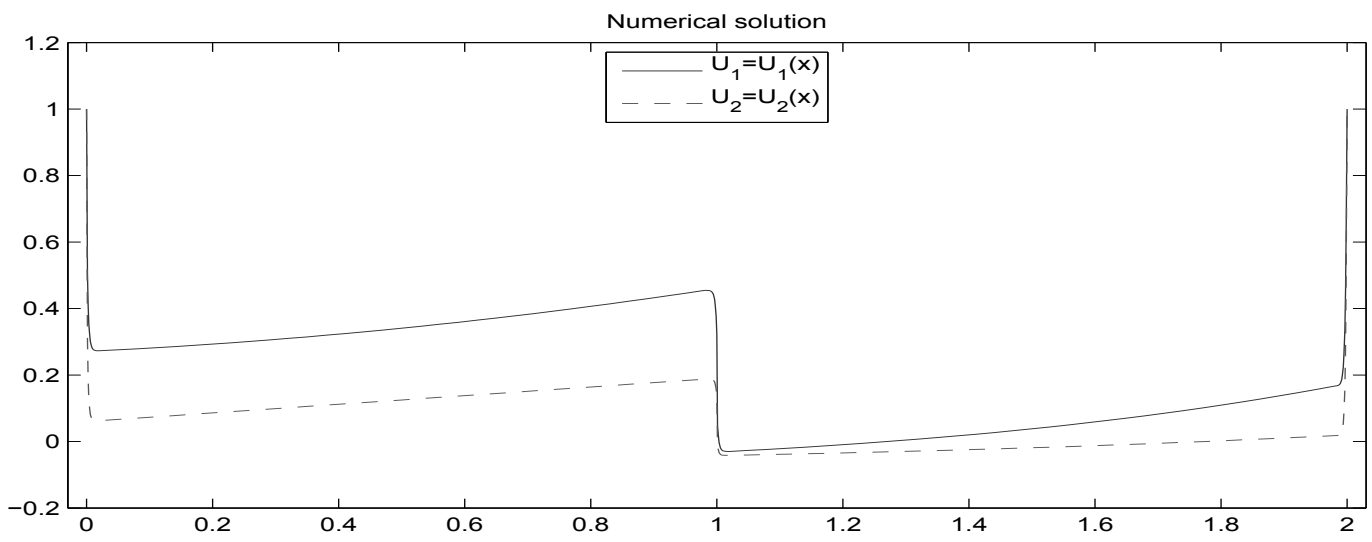

FIGURE 2. Numerical solution of the above Example 8.2.

TABLE 1. Numerical Results for the Example 8.1

\begin{tabular}{|l|c|c|c|c|c|c|c|}
\hline \multicolumn{9}{|c|}{ M (Number of mesh points) } \\
\hline & 32 & 64 & 128 & 256 & 512 & 1024 & 2048 \\
\hline \hline$D_{1}^{M}$ & $2.8268 \mathrm{e}-2$ & $1.2121 \mathrm{e}-2$ & $4.2829 \mathrm{e}-3$ & $1.5128 \mathrm{e}-3$ & $5.0124 \mathrm{e}-4$ & $1.5237 \mathrm{e}-4$ & $4.7635 \mathrm{e}-5$ \\
\hline$p_{1}^{M}$ & 1.2217 & 1.5008 & 1.5013 & 1.5937 & 1.7179 & 1.6774 & - \\
\hline \hline$D_{2}^{M}$ & $4.4596 \mathrm{e}-2$ & $2.0012 \mathrm{e}-2$ & $7.3012 \mathrm{e}-3$ & $2.5038 \mathrm{e}-3$ & $8.5338 \mathrm{e}-4$ & $2.6074 \mathrm{e}-4$ & $8.0558 \mathrm{e}-5$ \\
\hline$p_{2}^{M}$ & 1.1560 & 1.45477 & 1.5440 & 1.5529 & 1.7106 & 1.6945 & - \\
\hline
\end{tabular}

TABLE 2. Numerical Results for the Example 8.2

\begin{tabular}{|l|c|c|c|c|c|c|c|}
\hline \multicolumn{9}{|c|}{ M (Number of mesh points) } \\
\hline & 64 & 128 & 256 & 512 & 1024 & 2048 & 4096 \\
\hline \hline$D_{1}^{M}$ & $2.5288 \mathrm{e}-2$ & $8.9355 \mathrm{e}-3$ & $3.1562 \mathrm{e}-3$ & $1.0457 \mathrm{e}-3$ & $3.1788 \mathrm{e}-4$ & $9.9382 \mathrm{e}-5$ & $2.9950 \mathrm{e}-5$ \\
\hline$p_{1}^{M}$ & 1.5008 & 1.5013 & 1.5937 & 1.7179 & 1.6774 & 1.7304 & - \\
\hline \hline$D_{2}^{M}$ & $4.1935 \mathrm{e}-2$ & $1.5299 \mathrm{e}-2$ & $5.2467 \mathrm{e}-3$ & $1.7882 \mathrm{e}-3$ & $5.4636 \mathrm{e}-4$ & $1.6880 \mathrm{e}-4$ & $5.1480 \mathrm{e}-5$ \\
\hline$p_{2}^{M}$ & 1.4547 & 1.5440 & 1.5529 & 1.7106 & 1.6945 & 1.7133 & - \\
\hline
\end{tabular}

\section{DisCUSSION}

A class of BVPs for one type of SPDDEs of reaction- diffusion type are considered. To obtain an approximate solution for this type of problems, a numerical method named as initial value method (IVM) is suggested. The method is shown to be of order $O\left(N^{-2} \log ^{2} N\right)$, that is, the method has almost second order convergence. This is very much reflected in the numerical results presented in the Tables 1 and 2. Figures 1 and 2 represent the numerical solutions of the problems stated in Examples 8.1 and 8.2. From the Figures 1 and 2, it is obvious that, the 
solutions of the problems (8.1) and (8.2) exhibit strong boundary layers at $x=0, x=1$ and $x=2$. But this is not the case for convection diffusion type problems and in fact there is a boundary layer only at $x=2$ [19]. The present method works irrespective of the fact that the source term is continuous or not.

\section{REFERENCES}

[1] Thomas Erneux, Applied Delay Differential Equations. Springer (2009)

[2] A. Longtin and J. G. Milton, Complex oscillations in the human pupil light reflex with mixed and delayed feedback. Math. Biosci., 90 (1988), 183-199.

[3] C. G. Lange and R.M. Miura, Singular perturbation analysis of boundary-value problems for differentialdifference equations V. Small shifts with layer behavior. SIAM. J. APPL. MATH., 54(1) (1994), 249-272.

[4] M.W. Derstine, H.M. Gibbs, F.A. Hopf and D.L. Kaplan, Bifurcation gap in a hybrid optical system. Phys. Rev. A., 26 (1982), 3720-3722.

[5] V.Y. Glizer, Asymptotic analysis and solution of a finite-horizon $H_{\infty}$ control problem for singularly-perturbed linear systems with small state delay. J. Optim. Theory Appl., 117 (2003), 295-325.

[6] P.A. Farrell, A.F. Hegarty, J.J.H. Miller, E. O'Riordan and G. I. Shishkin, Robust computational techniques for boundary layers. Chapman Hall/ CRC, Boca Raton, (2000).

[7] E.P. Doolan, J.J.H. Miller and W.H.A. Schilders, Uniform numerical methods for problems with initial and boundary layers. Boole, Dublin, 1980.

[8] P.A. Farrell, J.J.H. Miller, E. O'Riordan and G. I. Shishkin, Singularly perturbed differential equations with discontinuous source terms. In: Analytical and Numerical Methods for Convection-Dominated and Singularly Perturbed Problems. (L.G. Vulkov, J.J.H. Miller and G.I. Shishkin eds.), pp 23-32, New York, USA : Nova Science Publishers. Inc, (2000).

[9] P.A. Farrell, A.F. Hegarty, J.J.H. Miller, E. O’Riordan and G.I. Shishkin, Singularly perturbed convection diffusion problems with boundary and weak interior layers. Journal of Computational Applied Mathematics, 166 (2004), 133-151.

[10] M. K. Kadalbajoo and K. K. Sharma, An $\varepsilon$-uniform fitted operator method for solving boundary-value problems for singularly perturbed delay differential equations: Layer behavior. International Journal of Computer Mathematics, 80(10) (2003), 1261-1276.

[11] M. K. Kadalbajoo and K. K. Sharma, Parameter uniform fitted mesh method for singularly perturbed delay differential equations with layer behavior. Electronic Transaction on Numerical Analysis, 23 (2006), 180-201.

[12] Pratima Rai and K. K. Sharma, Fitted mesh method for singularly perturbed delay differential turning point problems exhibiting boundary layers. International Journal of Computer Mathematics, 89(7) (2012), 944-961.

[13] M. K. Kadalbajoo and K. K. Sharma, Numerical Analysis of Boundary-Value Problems for SingularlyPerturbed Differential- Difference Equations with Small Shifts of Mixed Type. Journal of Optimization Theory and Applications, 115(1) (2002), 145-163.

[14] J. Mohapatra and S. Natesan, Uniformly convergent numerical method for singularly perturbed differentialdifference equation using grid equidistribution. International Journal for Numerical Methods in Biomedical Engineering, 27(9) (2011), 1427-1445.

[15] M. K. Kadalbajoo and K. K. Sharma, A numerical method based on finite difference for boundary value problems for singularly perturbed delay differential equations. Applied Mathematics and Computation, 197 (2008), 692-707.

[16] M. K. Kadalbajoo and K. K. Sharma, Parameter uniform numerical method for a boundary value problem for singularly perturbed nonlinear delay differential equation of neutral type. International Journal of Computer Mathematics, 81(7) (2004), 845-862.

[17] M.K. Kadalbajoo and Devendra Kumar, A computational method for singularly perturbed nonlinear differential-difference equations with small shift. Applied Mathematical Modelling, 34 (2010), 2584-2596. 
[18] V. Subburayan and N. Ramanujam, Asymptotic Initial Value Technique for singularly perturbed convectiondiffusion delay problems with boundary and weak interior layers. Applied Mathematics Letters, 25 (2012), 2272-2278.

[19] V. Subburayan and N. Ramanujam, An Initial Value Technique for Singularly Perturbed Convection-Diffusion Problems with a Delay. Journal of Optimization Theory and Applications, 158(1) (2013), 234-250.

[20] C. G. Lange and R. M. Miura, Singular perturbation analysis of boundary-value problems for differentialdifference equations. SIAM. J. APPL. MATH., 42(3) (1982), 502-530.

[21] C. M. Bender and S. A. Orszag, Advanced Mathematical Methods for Scientists and Engineers. McGraw-Hill Book Co. Singapore, (1984).

[22] Zhongdi Cen, A Hybrid finite difference scheme for a class of singularly perturbed delay differential equations. Neural, Parallel, and Scientific Computations, 16 (2008), 303-308.

[23] Zhongdi Cen, Jingfeng Chen and Lifeng Xi, A Second-Order Hybrid Finite Difference Scheme for a system of Coupled Singularly Perturbed Initial Value Problems. International Journal of Nonlinear Science, 8(2) (2009), $148-154$.

[24] T. Valanarasu and N. Ramanujam, An asymptotic Initial-Value Method for boundary value problems for a system of singularly perturbed Second-Order ordinary differential equations. Applied Mathematics and Computation, 147 (2004), 227-240. 